\title{
Esterificação do ácido oleico utilizando aluminossilicato mesoporoso modificado com óxido de zircônio
}

\author{
Esterification of oleic acid using mesoporous \\ aluminosilicate modified with zirconium \\ oxide
}

\author{
Bruna Ezequielle Bernardes Costa ${ }^{1}$, Fernando Cesário Rangel ${ }^{1}$, \\ Rosenira Serpa da Cruz ${ }^{1}$
}

\footnotetext{
${ }^{1}$ Grupo Bioenergia e Meio Ambiente - Universidade Estadual de Santa Cruz, Campus Soane Nazaré de Andrade, Rodovia Jorge Amado, Bairro Salobrinho, CEP: 45662-900, Ilhéus, Bahia, Brasil.

e-mail: brunabernardes18@hotmail.com, fcrangel@gmail.com, roserpa@uesc.br
}

\section{RESUMO}

Os desafios decorrentes do aumento populacional, do uso extensivo e degradação dos recursos naturais e da dependência dos recursos fósseis, tem despertado o interesse pelo desenvolvimento de tecnologias para a produção de combustíveis renováveis. Dentro deste contexto tecnologias para a produção de biodiesel tem sido bastante estudadas de forma a aliar economicidade e redução de impactos ambientais. A esterificação utilizando catalisador heterogêneo ácido possibilita o aproveitamento de matérias-primas de baixo valor agregado e redução de operações unitárias para separação e purificação dos produtos. Dentro destas premissas, o presente trabalho teve como objetivo sintetizar e caracterizar o material AlSBA-15, na razão Si/Al=10, modificada com $\mathrm{ZrO}_{2}$ e avaliá-lo como catalisador heterogêneo na esterificação do ácido oleico com metanol para a produção de ésteres monoalquílicos. A síntese da AlSBA-15, baseou-se na adição de um surfactante direcionador de estrutura Pluronic-123, TEOS e isopropóxido de alumínio. Para a modificação da AlSBA-15 realizou-se uma mistura física contendo $10 \%(\mathrm{~m} / \mathrm{m}) \mathrm{ZrO}_{2}$ em relação ao suporte, seguido de tratamento térmico. A caracterização do catalisador foi feita por difração de raios X, espectrometria na região do infravermelho, adsorção - dessorção de nitrogênio a $-196{ }^{\circ} \mathrm{C}$ e quantificação de acidez de Brönsted-Lowry. O desempenho catalítico foi avaliado em um reator Parr de $300 \mathrm{~mL}$, com razão metanol: ácido oleico igual a 6:1, 3\% $\mathrm{m} / \mathrm{m}$ de catalisador em relação ao ácido oleico, $3 \mathrm{~h}$ de reação à temperatura de $120{ }^{\circ} \mathrm{C}$. As taxas de conversão do ácido oleico (\%) foram, respectivamente, 55,5 $\pm 2,9,68,2 \pm 3,4$ e 79,8 \pm 3,6 para a, SBA-15, AlSBA-15 e a AlSBA-15- $\mathrm{ZrO}_{2}$, o que comprova a eficiência do método de síntese no aumento da força ácida do catalisador heterogêneo para a reação de esterificação. Os testes de lixiviação e de reutilização mostraram que a AlSBA-15- $\mathrm{ZrO}_{2}$ é ativa e estável nas condições reacionais empregadas.

Palavras-chave: Catálise heterogênea, esterificação, aluminosilicato mesoporoso, óxido de zircônio.

\begin{abstract}
Challenges stemming from population growth, extensive use and degradation of natural resources and dependence on fossil resources have aroused interest in the development of technologies for the production of renewable fuels. Within this context technologies for the production of biodiesel have been well studied in order to combine economicity and reduction of environmental impacts. Esterification using heterogeneous acid catalyst enables the use of raw materials of low added value and reduction of unit operations for separation and purification of products. The objective of the present work was to synthesize and characterize the AlSBA-15 material in the $\mathrm{Si} / \mathrm{Al}=10$ ratio modified with $\mathrm{ZrO} 2$ and to evaluate it as a heterogeneous catalyst in the esterification of oleic acid with methanol for the production of monoalkyl esters. The characterization of the catalyst was done by X - ray diffraction, infrared spectrometry, nitrogen adsorption-desorption at -196 ${ }^{\circ} \mathrm{C}$ and Brönsted - Lowry acid quantification. The catalytic performance was evaluated in a $300 \mathrm{~mL}$ Parr reactor, with methanol/oleic acid ratio equal to $6 / 1$, with $3 \% \mathrm{~m} / \mathrm{m}$ of catalyst in relation to oleic acid and $3 \mathrm{~h}$ of
\end{abstract}


reaction at $120^{\circ} \mathrm{C}$. Conversion rates of oleic acid (\%) were, respectively, 55.5 $\pm 2.9,68.2 \pm 3.4$ and $79.8 \pm$ 3.6 for SBA-15, AlSBA-15 and AlSBA -15-ZrO2, which proves the efficiency of the synthesis method in increasing the acidic strength of the heterogeneous catalyst for the esterification reaction. The leaching and reuse tests showed that AlSBA-15-ZrO2 is active and stable under the reaction conditions employed.

Keywords: Heterogeneous catalyst, esterification, mesoporous aluminosilicate, zirconium oxide.

\section{INTRODUÇÃO}

A matriz energética mundial é pautada majoritariamente em recursos fósseis, no entanto as crescentes preocupações com as questões ambientais bem com a busca por menor dependência externa por recursos fósseis têm estimulado a busca por fontes renováveis de energia. Nesse cenário o biodiesel tem se destacado por apresentar diversas vantagens, dentre elas a redução dos níveis de poluição ambiental e compatibilidade com a tecnologia dos motores do ciclo-diesel [1,2].

O biodiesel é majoritariamente produzido por transesterificação via catálise alcalina, em meio homogêneo. Porém, para que essa rota de produção seja tecnicamente viável se faz necessária a utilização de óleo com baixo teor de ácido graxo livre (menos que $1 \%$ de acidez) e umidade (todos os reagentes empregados devem ser anidros), o que representa cerca de $80 \%$ do custo total da produção de biodiesel, além de necessitar de intensas etapas de lavagem para purificação do produto, neutralização do catalisador e recuperação da glicerina [3]. Devido a esses inconvenientes, mesmo com o processo industrial já estabelecido, nos últimos anos tem se observado um crescente aumento nos trabalhos acadêmicos e industriais voltados para o desenvolvimento e otimização dos processos de produção de biodiesel [2-7].

Pesquisas de novos processos consistem em processar qualquer matéria-prima, independente de sua origem (gordura animal, óleo vegetal, residual ou não), em ésteres monoalquílicos. Neste cenário, a produção de biodiesel, via a catálise ácida, é uma opção promissora uma vez que permite a obtenção de ésteres monoalquílicos tanto por reações de esterificação dos ácidos graxos, quanto por reações de transesterificação dos triacilgliceróis ou ainda hidroesterificação, o que possibilita a utilização de matérias-primas de baixo custo e, portanto, mais viáveis economicamente [5].

A esterificação utilizando catalisadores ácidos tem se destacado por aliar as vantagens técnicas e ambientais associadas a catálise heterogênea quando comparada com a catálise homogênea bem como por ser uma reação que permite uma versatilidade de rotas tecnológicas, podendo ser utilizada como pré-etapa à transesterificação, simultânea à transesterificação ou ainda como reação única para a obtenção de ésteres monoalquílicos [2,5].

Muitas têm sido as propostas de sólidos para utilização como catalisadores para a produção do biodiesel. Dentre os diversos sólidos, o grupo das peneiras moleculares tem apresentado grande destaque, pois demonstram a capacidade de associar as propriedades químicas, espaciais e mecânicas necessárias e possibilidade de funcionalização da superfície para ajuste de propriedades [8-11].

Diante do exposto, este trabalho teve como objetivo sintetizar e caracterizar materiais do tipo AlSBA15 modificado com $\mathrm{ZrO}_{2}$ para o uso como catalisador heterogêneo na esterificação de ácido oleico com metanol. A avaliação da estabilidade frente à lixiviação das espécies ativas para o meio reacional bem como a capacidade de reutilização também foram avaliadas.

\section{MATERIAIS E MÉTODOS}

\subsection{Síntese dos materiais}

Para a síntese de SBA-15 foi utilizada uma síntese verificada proposta por MEYNEN et al.[12], utilizando-se os reagentes nas seguintes razões molares 0,017TEOS: 5,87P123: $194 \mathrm{HCl}: 208 \mathrm{H}_{2} \mathrm{O}$. Primeiro foram misturados à água destilada, o ácido clorídrico e o $\mathrm{P} 123 \mathrm{em}$ béquer de polipropileno a $45^{\circ} \mathrm{C}$ sob agitação até a dissolução completa do surfactante. Em seguida, foi adicionado o TEOS, permanecendo, sob agitação, durante 7,5 $\mathrm{h}$ a $45^{\circ} \mathrm{C}$. Cessada a agitação, a mistura foi aquecida a $80^{\circ} \mathrm{C}$ por $15,5 \mathrm{~h}$. O material foi filtrado, lavado com água destilada e etanol e seco a $100{ }^{\circ} \mathrm{C}$ por $2 \mathrm{~h}$ em uma estufa. Por fim, o material foi calcinado em mufla a $550{ }^{\circ} \mathrm{C}$ durante $6 \mathrm{~h}$ em uma taxa de aquecimento de $1{ }^{\circ} \mathrm{C} \min ^{-1}$.

Um procedimento experimental semelhante foi utilizado para a síntese da Al-SBA-15. No entanto, juntamente com o TEOS, foi acrescentado o isopropóxido de alumínio (iPrAl) como fonte de alumínio na razão molar $\mathrm{Si} / \mathrm{Al}=10$. Para esta síntese foram utilizados os reagentes nas proporções molares de 0,1TEOS: 0,017 iPrAl: 5,87 P123: $194 \mathrm{HCl}: 208 \mathrm{H}_{2} \mathrm{O}$. 
Para a impregnação da AlSBA-15 com $10 \%(\mathrm{~m} / \mathrm{m}) \mathrm{ZrO}_{2}$, realizou-se uma mistura física de $0,25 \mathrm{~g}$ de AlSBA-15 e 0,025 g do óxido de zircônio IV com o auxílio do grau de ágata. Em seguida, a mistura física foi submetida ao seguinte tratamento térmico (taxa de aquecimento de $5^{\circ} \mathrm{C} \mathrm{min}^{-1}$ e fluxo de nitrogênio): da temperatura ambiente até $100^{\circ} \mathrm{C}$ e permanência nesta temperatura por 30 minutos, em seguida, a temperatura foi elevada até $250{ }^{\circ} \mathrm{C}$, sendo mantida por 30 minutos, depois até $350{ }^{\circ} \mathrm{C}$ permanecendo nesta temperatura por mais 30 minutos e por fim, até $500{ }^{\circ} \mathrm{C}$ permanecendo nesta temperatura por $1 \mathrm{~h}$. Esta amostra será denominada AlSBA-15- $\mathrm{ZrO}_{2}$.

\subsection{Caracterização dos materiais}

O difratograma de raios X (DRX) foi obtido em um difratômetro, marca Siemens, modelo D5000, utilizando como fonte de radiação o $\mathrm{CuK} \alpha$. A região analisada foi de $2 \theta$ de $0,5^{\circ}$ a $10^{\circ}$ (difração a ângulos baixos), em uma velocidade de $4^{\circ} \mathrm{s}^{-1}$.

As isotermas de adsorção-dessorção de nitrogênio foram obtidas a $-196{ }^{\circ} \mathrm{C}$ usando um aparelho Micromeritics ASAP 2010. A amostra foi previamente tratada a $250{ }^{\circ} \mathrm{C}$, sob fluxo de nitrogênio, por $17 \mathrm{~h}$. O diâmetro médio de poro bem como a distribuição de tamanho de poros foram calculadas a partir da isoterma de adsorção de nitrogênio usando o método BJH (Barret Joyner Halenda) para sólidos microporosos. A área específica foi calculada usando a equação BET na região de baixa pressão $(\mathrm{p} / \mathrm{po}=0,200)$ e o volume de microporo usou o método t-plot.

Os espectros (FTIR) foram obtidos com um espectrofotômetro marca Shimadzu com faixa de 4000 a $400 \mathrm{~cm}^{-1} \mathrm{e}$ uma resolução de $4 \mathrm{~cm}^{-1}$. As amostras foram preparadas na forma de pastilha de $\mathrm{KBr}$ com uma proporção de $1 \%$ da amostra.

O teste de acidez do catalisador consistiu na determinação por titulação do número de sítios ácidos de Brönsted-Lowry. Em resumo, $100 \mathrm{mg}$ do material foram deixados em contato com $20 \mathrm{~mL}$ de $\mathrm{NaOH}$ 0,1 mol $\mathrm{L}^{-1}$, por $3 \mathrm{~h}$, sob leve agitação. Após esse período, foram retiradas alíquotas da solução básica que foram tituladas com $\mathrm{HCl} 0,1 \mathrm{~mol} \mathrm{~L}^{-1}$, para se verificar a quantidade de $\mathrm{NaOH}$ que reagiu com o material. A acidez foi determinada em mmol de $\mathrm{H}^{+}$por grama de material.

\subsection{Reação de esterificação do ácido oléico com metanol}

Os materiais foram avaliados frente à reação de esterificação do ácido oleico com metanol em um reator Parr de $300 \mathrm{~mL}$, empregando as seguintes condições reacionais: razão metanol: ácido oléico igual a 6:1, com 3\% $\mathrm{m} / \mathrm{m}$ de catalisador em relação ao ácido oléico, em $3 \mathrm{~h}$ de reação à temperatura de $120^{\circ} \mathrm{C}$. O monitoramento da reação foi feito através da determinação da acidez segundo o método ABNT NBR 14448 (titulação potenciométrica) em um titulador automático (Titrino 798 MPT). Os produtos foram filtrados para a retirada do catalisador, lavados e secos.

Para verificar a ocorrência ou não de lixiviação das espécies ativas para o meio reacional, foi preparada uma reação padrão e após 30 minutos, foi realizada a retirada do catalisador por filtração na mesma temperatura da reação, e a porção filtrada foi colocada a $120^{\circ} \mathrm{C}$ por mais $2,5 \mathrm{~h}$ e, então foram retiradas alíquotas para análise da acidez.

A capacidade de reutilização é um dos fatores mais críticos para a que um material possa ser aplicado como catalisador heterogêneo na indústria. Após 3 horas de reação o catalisador foi lavado com metanol, seguido de secagem em estufa a $120^{\circ} \mathrm{C}$ por 2 horas. Em seguida reutilizado em um novo ciclo de reação mantendo-se as proporções utilizadas no procedimento padrão. Foram realizados ao todo cinco ciclos com o mesmo catalisador.

\section{RESULTADOS E DISCUSSÃO}

\subsection{Caracterização dos materiais}

Na Figura 1 são apresentados os espectros na região do infravermelho das amostras SBA-15, AlSBA-15 e AlSBA-15- $\mathrm{ZrO}_{2}$. A Tabela 1 resume dos dados relativos às frequências vibracionais observadas e suas respectivas atribuições para o espectro de infravermelho que confirma os principais grupos característicos dos silicatos e aluminosilicatos [13]. 


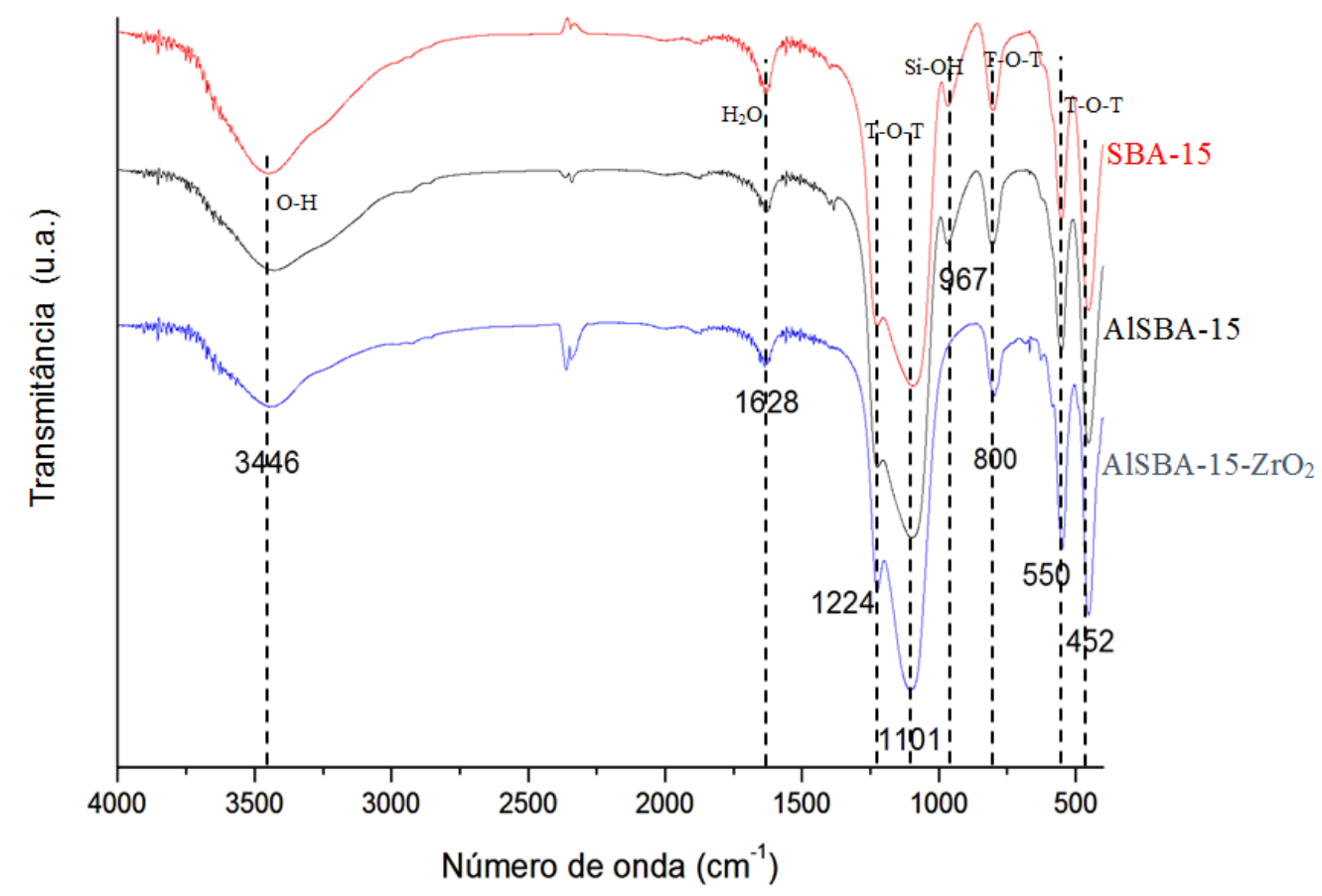

Figura 1: Espectroscopia na região do Infravermelho (FTIR) das amostras SBA15, AlSBA15 e Al-SBA-15 e Al-SBA$15-\mathrm{ZrO}_{2}$.

Enquanto que, a Tabela 1 resume os dados relativos às frequências vibracionais observadas e suas respectivas atribuições no espectro de infravermelho, confirmando os principais grupos característicos dos silicatos e aluminosilicatos [13].

Tabela 1: Dados relativos às frequências vibracionais observadas e suas respectivas atribuições feitas para o espectro de infravermelho da amostra do suporte AlSBA-15[13,14]

\begin{tabular}{|c|c|}
\hline Número de onda $\left(\mathrm{cm}^{-1}\right)$ & Atribuições \\
\hline $3400-3500$ & $\begin{array}{c}\text { Estiramento dos grupos hidroxilas internos e externos } \\
\text { na estrutura mesoporosa, e as moléculas de água de } \\
\text { hidratação. }\end{array}$ \\
\hline $1620-1630$ & Água interagindo com a superfície do suporte. \\
\hline $1213-1081$ & $\begin{array}{l}\text { Estiramentos assimétricos da ligação T-O-T } \\
\qquad(\mathrm{T}=\mathrm{Si}, \mathrm{Al}) .\end{array}$ \\
\hline $950-970$ & $\begin{array}{l}\text { Vibrações Si-OH gerado pela presença de sítios defei- } \\
\text { tuosos na estrutura. }\end{array}$ \\
\hline 800 & $\begin{array}{l}\text { Estiramentos simétricos das ligações } \mathrm{T}-\mathrm{O}-\mathrm{T}(\mathrm{T}=\mathrm{Si} \text {, } \\
\qquad \mathrm{Al}) .\end{array}$ \\
\hline $450-470$ & Deformações da ligação T-O-T (T=Si, Al) \\
\hline
\end{tabular}

É importante ressaltar que a banda em torno de $950-970 \mathrm{~cm}^{-1}$ atribuída às vibrações Si-OH gerada pela presença de sítios defeituosos na estrutura, só foi observada na SBA-15 e AlSBA-15. Esta observação sugere que, sendo o silanol defeitoso mais reativo, este pode ser um indicativo que o óxido de zircônio foi incorporada ao material por interações do $\mathrm{ZrO}_{2}$ com estes grupos silanóis ( $\left.\mathrm{Si}-\mathrm{OH}\right)$ presentes na parte externa e/ou interna do suporte AlSBA-15 [14]. 
Não foram observadas as bandas na região entre $2960-2870 \mathrm{~cm}^{-1}$ que estão relacionadas ao estiramento entre a ligação $\mathrm{C}-\mathrm{H}$ dos grupos $\mathrm{CH}_{2}$ das moléculas orgânicas do direcionador, as bandas referentes às deformações da ligação $-\left(\mathrm{CH}_{2}\right) \mathrm{n}$ da cadeia em $1460 \mathrm{~cm}^{-1}$ e a banda referente a ligação C-O-C do direcionador Pluronic P123 em $1390 \mathrm{~cm}^{-1}$, o que comprova a extração efetiva do direcionador Pluronic P123 durante o tratamento térmico. Esta remoção completa é importante para evitar o bloqueio dos poros com consequente prejuízo à impregnação do óxido de zircônio e à atividade catalítica do material $[15,16]$.

Na Figura 2 são apresentados os padrões de difração de raios X para as amostras SBA-15, AlSBA-15 e AlSBA-15- $\mathrm{ZrO}_{2}$. Observa-se que os todos os materiais sintetizados apresentam três picos de difração referentes aos planos de difração com índices de Miller (100), (110) e (200), característicos da estrutura mesoporosa bidimensional com simetria P6mm, mostrando que a síntese dos materiais foi bem sucedida [17].

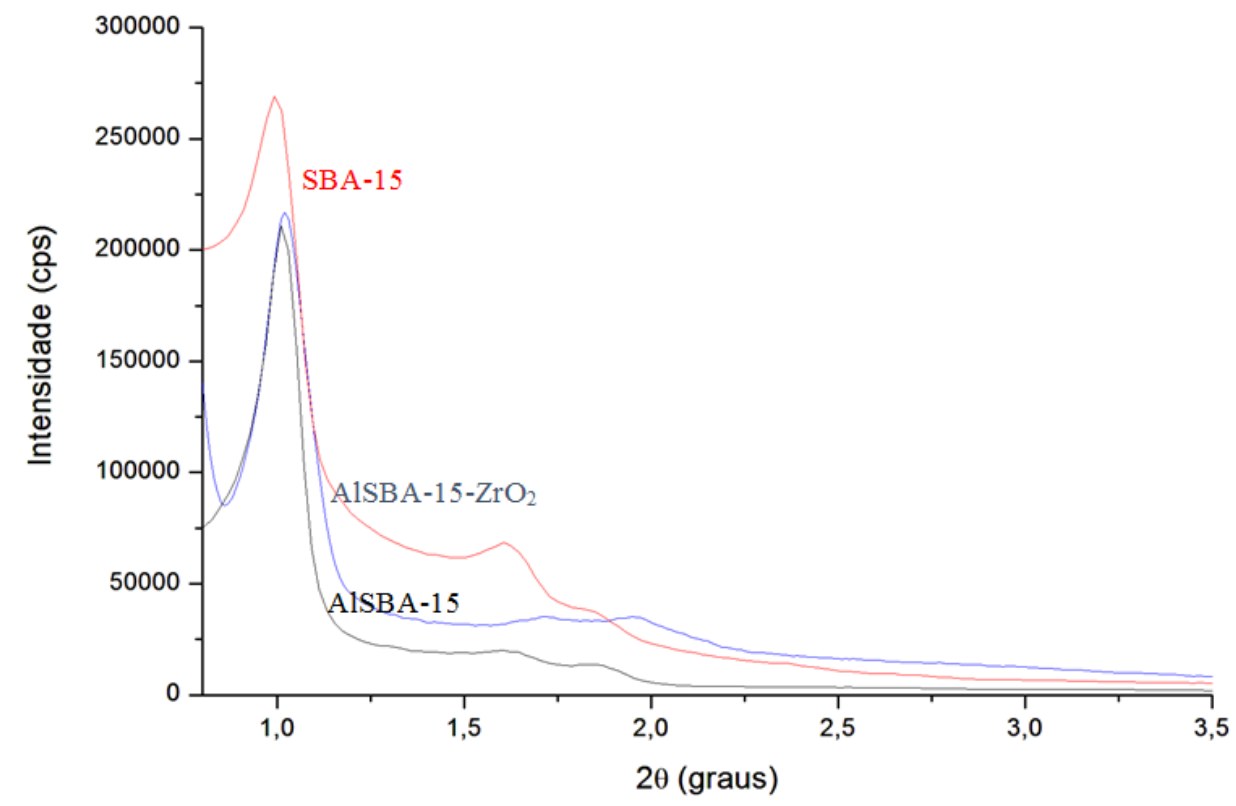

Figura 2: Padrão de difração de raios-X das amostras.

Esta é uma evidência de que mesmo após a modificação da SBA-15 com $\mathrm{ZrO}_{2}$, o ordenamento da estrutura mesoporosa não foi comprometido além disso, as paredes de sílica não foram afetadas. Quanto à redução da intensidade dos picos de difração no material AlSBA-15- $\mathrm{ZrO}_{2}$, sabe-se que geralmente a impregnação de óxidos metálicos nos poros de materiais mesoporosos do tipo SBA-15, podem promover certa distorção nos canais mesoporosos devido à interação destes óxidos com os grupos funcionais presentes nas paredes internas de seus poros e isto normalmente reflete em um alargamento e deslocamento da maiores valores de 2e do pico referente ao plano (110) nos padrões de difração de raios $\mathrm{X}$ desses materiais $[9,18]$.

As isotemas de adsorção - dessorção de nitrogênio a $-196{ }^{\circ} \mathrm{C}$ e a distribuição de diâmetros de poros estão mostradas na Figura 3. Os resultados área específica, volume total e diâmetro médio de poros obtidos por adsorção - dessorção de nitrogênio e do parâmetro de rede e espessura da parede, calculados a partir dos dados de difração de raios X estão apresentados na Tabela 2. 

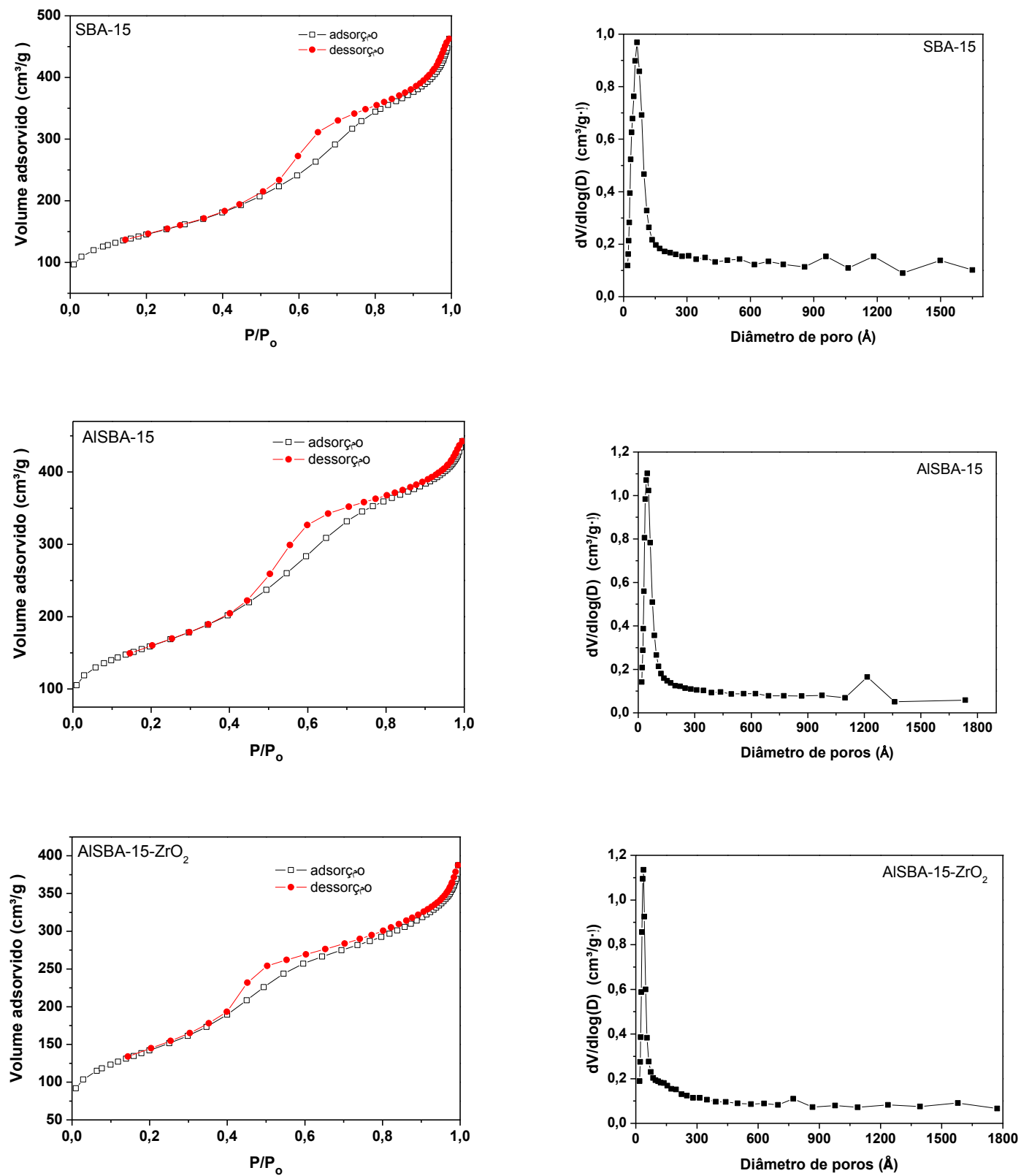

Figura 3: Isotermas de adsorção-dessorção de $\mathrm{N}_{2}$ e curvas de distribuição de diâmetro de poros para os materiais SBA15, AlSBA-15 e AlSBA-15- $\mathrm{ZrO}_{2}$

As isotermas indicam que todas as amostras apresentaram isotermas do tipo IV, o que confirma a mesoporosidade dos materiais, com histerese do tipo I que são também características de materiais mesoporosos com sistema de poros cilíndricos, ou feitos a partir de agregados ou aglomerados de partículas esferoidais com poros de tamanhos uniformes [16]. 
Tabela 2: Área específica, diâmetro e volume médio de poros e volume total de poros dos materiais sintetizados.

\begin{tabular}{cccccc}
\hline Material & $\begin{array}{c}\text { Área específi- } \\
\text { ca BET } \mathbf{~ m}^{\mathbf{2}} \mathbf{g}-\end{array}$ & $\begin{array}{c}\text { Volume } \\
\text { total de } \\
\text { poros } \\
\left(\mathbf{c m}^{\mathbf{3}} \mathbf{g}^{-\mathbf{1}}\right)\end{array}$ & $\begin{array}{c}\text { Diâmetro médio } \\
\text { de poro BJH } \\
(\mathbf{n m})\end{array}$ & $\begin{array}{c}\text { Parâmetro de rede } \\
\text { da estrutura porosa } \\
*(\mathbf{n m})\end{array}$ & $\begin{array}{c}\text { Espessura mé- } \\
\text { dia da parede** } \\
(\mathbf{n m})\end{array}$ \\
\hline SBA-15 & 513 & 0,65 & 4,63 & 10,3 & 5,67 \\
\hline AISBA-15 & 565 & 0,67 & 5,25 & 10,6 & 5,36 \\
\hline $\begin{array}{c}\text { AISBA- } \\
\mathbf{1 5 - Z r O 2 ~}\end{array}$ & 507 & 0,56 & 4,39 & 10,9 & 6,51 \\
\hline
\end{tabular}

$* a=\frac{2 d_{100}}{\sqrt{3}} \quad * * e=a+d p$. Dados obtidos a partir do padrão de difração de raios $\mathrm{X}$.

Quando comparamos os dados mostrados na Tabela 2 para a SBA-15 com os catalisador AlSBA-15$\mathrm{ZrO}_{2}$ observa-se diminuição nos valores das propriedades texturais e uma aumento das propriedades estruturais como o parâmetro de rede e a espessura da parede. Isto pode ser atribuído à modificação da superfície da SBA-15 com o óxido de zircônio que causou o bloqueio local dos canais porosos, dificultando a difusão do $\mathrm{N}_{2}$ até os sítios ativos presentes no interior dos poros, refletindo nos valores das propriedades texturais. $\mathrm{O}$ aumento das propriedades estruturais também corrobora com a interação do óxido de zircônio com a estrutura da AlSBA-15 devido ao maior comprimento da ligação Zr-O-Si quando comparada com a ligação Si-O-Si ou Si-O-Al bem como dispersão do óxido de zircônio nas paredes da AlSBA-15 [11,17-21]. Observa-se ainda que o material AlSBA-15- $\mathrm{ZrO}_{2}$ apresentou a distribuição mais estreita de diâmetro de poros corroborando com a dispersão do óxido na superfície do suporte.

\subsection{Reação de esterificação do ácido oléico com metanol}

Os resultados obtidos frente às reações de esterificação do ácido oleico bem como da quantificação dos sítios ácidos de Brönsted-Lowry para os materiais, estão dispostos na Tabela 3.

Tabela 3: Dados relativos às conversões do ácido oleico e quantificação de sítios ácidos de Brönsted-Lowry

\begin{tabular}{ccc}
\hline Materiais & $\begin{array}{c}\text { Sítios ácidos de Bronsted } \\
\left(\mathbf{m m o l ~ H} \mathbf{~ H}_{\mathbf{c a t}}\right)^{*}\end{array}$ & Esterificação $(\%)^{* *}$ \\
\hline SBA-15 & $1,29 \pm 0,25$ & $55,5 \pm 2,9$ \\
AlSBA-15 & $1,81 \pm 0,14$ & $68,2 \pm 3,4$ \\
AlSBA-15- $\mathrm{ZrO}_{2}$ & $2,11 \pm 0,23$ & $79,9 \pm 3,6$ \\
\hline
\end{tabular}

*Medidas feitas em triplicata. **Condições reacionais: Razão metanol/ácido oleico $6: 1,3 \% \mathrm{~m} / \mathrm{m}$ de catalisador em relação ao ácido oleico, 3 h de reação e temperatura de $120{ }^{\circ} \mathrm{C}$. As reações foram conduzidas em triplicata.

A esterificação de ácidos graxos pode ser catalisada por sítios ácidos de $\mathrm{C}$ bem como de Lewis. Os resultados da Tabela 3 mostram um aumento significativo da acidez de Brönsted-Lowry com a inserção do alumínio, na rede da SBA-15, no entanto com a incorporação do zircônio não foi observado o mesmo comportamento. Contudo, a maior atividade catalítica apresentada por este último material pode ser atribuída ao aumento da força ácida certamente pelo aumento do número de sítios ácidos de Lewis, com a inserção do óxido de zircônio IV, quando comparada com a AlSBA-15 [10].

O mecanismo proposto para a esterificação de ácidos graxos usando ácidos de Lewis como catalisadores já está bem descrito na literatura. As moléculas de ácidos graxos são adsorvidas na superfície do catalisador resultando em um aumento na densidade de carga positiva no carbono carbonílico, atribuída à interação entre o par de elétrons do oxigênio carbonílico do ácido graxo e o metal presente na estrutura do catalisador, favorecendo o ataque nucleofílico do par de elétrons da hidroxila alcoólica levando à formação de um inter- 
mediário tetraédrico, seguida de eliminação de uma molécula de água e do monoéster graxo [22]. Além disso, a presença do alumínio na rede da SBA-15 foi também outro favor determinante para contribuir com esta acidez, pois com a carga negativa decorrente da substituição do silício por alumínio na estrutura da SBA-15, advinda de sua quarta ligação, faz com que o hidrogênio ligado ao grupo - OH (hidroxila) associado à ele torne-se mais ácido[10, 18, 23]. Experimentos de dessorção termoprogramada de moléculas básicas estão sendo conduzidos de forma a identificar e quantificar os sítios ácidos destes catalisadores.

Portanto, a maior atividade catalítica da AlSBA-15- $\mathrm{ZrO}_{2}$ pode ser atribuída à ao maior densidade de sítios ácidos de Brönsted-Lowry resultante da inserção do Al na rede bem como bem como da maior força ácida de Lewis resultante da dispersão do óxido de zircônio no suporte com grande área especifica aliada a maior acessibilidade aos sítios ativos internos e,ou externos devido a estrutura mesoporosa bidimensional do catalisador como mostrado nos dados de difração de raios X e adsorção - dessorção de nitrogênio a $-196{ }^{\circ} \mathrm{C}$.

A estabilidade frente à lixiviação das espécies ativas para o meio reacional é um dos principais desafios na área da catálise heterogênea em fase líquida, reação que favorece o arraste das espécies ativas para o meio reacional, o que se caracteriza como contribuição da catálise homogênea, levando à desativação do catalisador. Tal estudo merece destaque, uma vez que, essas características são muitas vezes negligenciadas pela maioria dos trabalhos $[24,25]$. Os resultados obtidos frente à reação de lixiviação para os materiais estão dispostos na Tabela 4.

Tabela 4: Teste de lixiviação para os materiais SBA-15, AlSBA-15 e AlSBA-15- $\mathrm{ZrO}_{2}$

\begin{tabular}{llll}
\hline Tempo (min.) & SBA-15(\%) & AISBA-15(\%) & AlSBA-15-ZrO $(\%)$ \\
\hline 30 (retirada do material) & $18,1 \pm 3,2$ & $24,8 \pm 3,8$ & $38,6 \pm 3,5$ \\
60 & $19,0 \pm 3,6$ & $25,4 \pm 3,2$ & $39,4 \pm 3,9$ \\
120 & $19,4 \pm 4,1$ & $25,6 \pm 4,5$ & $39,8 \pm 4,3$ \\
180 & $20,1 \pm 2,9$ & $26,5 \pm 4,2$ & $41,1 \pm 3,8$ \\
\hline
\end{tabular}

*Condições reacionais para esterificação: Razão metanol/ácido oleico 6:1, $3 \% \mathrm{~m} / \mathrm{m}$ de catalisador em relação ao ácido oleico, 3 h de reação e temperatura de $120{ }^{\circ} \mathrm{C}$. As reações foram conduzidas em triplicata.

De acordo com os resultados obtidos frente ao teste de lixiviação é possível afirmar que os materiais são estáveis nas condições reacionais avaliadas, uma vez que após a retirada do catalisador por filtração à quente, a conversão do ácido oleico cessa. Diante disso, foram feitas as avaliações da capacidade de reutilização do catalisador, onde foram realizadas 5 reações consecutivas com o mesmo. Os resultados são mostrados na Tabela 5 .

Tabela 5: Teste de reutilização para os materiais SBA-15, AlSBA-15 e AlSBA-15-ZrO 2

\begin{tabular}{llll}
\hline Ciclo & SBA-15(\%) & AlSBA-15(\%) & AlSBA-15-ZrO $(\%)$ \\
\hline 1 & $55,5 \pm 2,9$ & $68,2 \pm 3,4$ & $79,8 \pm 3,6$ \\
2 & $55,0 \pm 3,3$ & $67,4 \pm 3,4$ & $78,4 \pm 3,8$ \\
3 & $56,1 \pm 3,5$ & $68,6 \pm 4,3$ & $78,8 \pm 4,1$ \\
4 & $54,2 \pm 2,9$ & $66,5 \pm 4,2$ & $79,1 \pm 3,3$ \\
5 & $55,2 \pm 3,2$ & $68,1 \pm 4,1$ & $79,4 \pm 3,2$ \\
\hline
\end{tabular}

*Condições reacionais para esterificação: Razão metanol/ácido oleico 6:1, $3 \% \mathrm{~m} / \mathrm{m}$ de catalisador em relação ao ácido oleico, $3 \mathrm{~h}$ de reação e temperatura de $120^{\circ} \mathrm{C}$. As reações foram conduzidas em triplicata.

Os resultados apresentados pelo teste de reutilização corroboram com o apresentado pelo teste de lixiaviação, uma vez que que não foi observado queda significativa na conversão do ácido oleico de um ciclo para o outro para nenhum dos materiais estudados, podendo confirmar a estabilidae do catalisador hetorogêneo nas condições reacionais empregadas. Estes resultados estão de acordo com os encontrados por 
MELERO et al.[8] na transtesrificação do óleo de palma com alto teor de ácidos graxos livres usando a catalisador Zr-SBA-15 com as seguintes condições razão metanol: óleo igual a 45,8, 12,45\% m/m de catalisador em relação ao óleo de palma, $6 \mathrm{~h}$ de reação à temperatura de $209{ }^{\circ} \mathrm{C}$ corroborando com a alta estabilidade térmica e química destes materiais.

\section{CONCLUSÕES}

O material AlSBA-15- $\mathrm{ZrO}_{2}$ obtido por inserção do alumínio por síntese direta seguida de impregnação por mistura física do $\mathrm{ZrO}_{2}$ apresentou propriedades texturais e estruturais típicas de materiais mesoporosos ordenamento hexagonal dos mesoporos. A modificação da SBA-15 com alumínio e óxido de zircônio IV promoveu a acidez do material com a inserção de acidez de Brösnted-Lowry e de Lewis o que tornou o material um catalisador ativo e estável nas condições utilizadas na reação de esterificação do ácido oleico com metanol.

\section{AGRADECIMENTOS}

RSC e BEBC agradecem á FAPESB pelo suporte financeiro e pela bolsa de estudo, respectivamente. Ao CETENE - Centro de Tecnologias Estratégicas do Nordeste pela análise de adsorção - dessorção de $\mathrm{N}_{2}$ a $196^{\circ} \mathrm{C}$.

\section{BIBLIOGRAFIA}

[1] HASAN, M. M., RAHMAN M. M., "Performance and emission characteristics of biodiesel-diesel blend and environmental and economic impacts of biodiesel production: A review", Renewable and Sustainable Energy Reviews, v. 74, pp. 938-948, 2017.

[2] LOURINHO, G., BRITO, P., “Advanced biodiesel production technologies: novel developments”, Reviews in Environmental Science and BioTechnology, v. 14, pp. 287-316, 2015.

[3] BASKAR, G., AISWARYA, R., "Trends in catalytic production of biodiesel from various feedstocks", Renewable and Sustainable Energy Reviews, v. 57, pp. 496-504, 2016.

[4] DANTAS, J., LEAL, E., MAPOSSA, A.B. et al., "Síntese, caracterização e performance catalítica de nanoferritas mistas submetidas a reação de transesterificação e esterificação via rota metílica e etílica para biodiesel", Matéria (R.J.), v.21, pp. 1080-1093, 2016.

[5] MARDHIAH, H. H., ONG, H. C., MASJUKI, H. H., et al. "A review on latest developments and future prospects of heterogeneous catalyst in biodiesel production from non-edible oils", Renewable and Sustainable Energy Reviews, v. 67, pp. 1225-1236, 2017.

[6] GO, A. W., SUTANTO, S., ONG, L. K., et al., "Developments in in-situ (trans) esterification for biodiesel production: A critical review", Renewable and Sustainable Energy Reviews, v. 60, pp. 284-305, 2016.

[7] WHEELDON, I. P. C., CHRISTOPHER, P., BLANCH, H. "Integration of heterogeneous and biochemical catalysis for production of fuels and chemicals from biomass", Current Opinion in Biotechnology, v. 45, pp. 127-135, 2017.

[8] LIANG, C., WEI, M. C., TSENG, H. H., et al. "Synthesis and characterization of the acidic properties and pore texture of Al-SBA-15 supports for the canola oil transesterification", Chemical engineering journal, v.223 pp.785-794, 2013.

[9] IGLESIAS, J., MELERO, J.A., BAUTISTA, L.F. et al., "Zr-SBA-15 as an efficient acid catalyst for FAME production from crude palm oil”, Catalysis Today, v.167, pp. 46-55, 2011.

[10] MELERO, J.A., IGLESIAS, J., BAUTISTA, L.F. et al., "Zr-SBA-15 acid catalyst: Optimization of the synthesis and reaction conditions for biodiesel production from low-grade oils and fats", Catalysis Today, v.195. pp. 44-53, 2012.

[11] CABRERA-MUNGUIA, D. A., GONZÁLEZ, H., GUTIÉRREZ-ALEJANDRE, A., "Heterogeneous acid conversion of a tricaprylin-palmitic acid mixture over Al-SBA-15 catalysts: Reaction study for biodiesel synthesis", Catalysis Today, v.282, pp. 195-203, 2017.

[12] MEYNEM, V., COOL, P., VANSANT, E. F. "Verified syntheses of mesoporous materials", Microporous and mesoporous materials, v.125.3, pp. 170-223, 2009. 
[13] LEE, W.K.W., VAN DEVENTER, J.S.J. "Use of infrared spectroscopy to study geopolymerization of heterogeneous amorphous aluminosilicates", Langmuir, v.19, n.21, pp.8726-8734, 2003.

[14] DO, D.M., JAENICKE, S., CHUAH , G-K, "Mesoporous Zr-SBA-15 as a green solid acid catalyst for the Prins reaction”, Catalysis Science \& Technology, v. 2, n.7, pp. 1417-1424, 2012.

[15] JIANG, Q., WU, Z. Y., WANG, Y. M., et al. "Fabrication of photoluminescent ZnO/SBA-15 through directly dispersing zinc nitrate into the as-prepared mesoporous silica occluded with template", Journal of Materials Chemistry, v.16, pp. 1536-1542, 2006.

[16] CHAUDHURI, H. S. D.; ASHIS S. "SBA-15 functionalised with high loading of amino or carboxylate groups as selective adsorbent for enhanced removal of toxic dyes from aqueous solution", New Journal of Chemistry, v.40, pp. 3622-3634, 2016.

[17] ZHAO, D., FENG, J., HUO, Q., et al. "Triblock copolymer syntheses of mesoporous silica with periodic 50 to 300 angstrom pores”, Science, v.279. n. 5350, pp. 548-552,1998.

[18] IGLESIAS , J., MELERO, J.A., MORALES, G., et al.,"Zr-SBA-15 Lewis Acid Catalyst: Activity in Meerwein Ponndorf Verley Reduction”, Catalysts, v. 5, pp.1911-1927, 2015.

[19] EL-NAHHAL, I. M., SALEM, J. K., SELMANE, M., et al., "Synthesis and structural characterization of $\mathrm{ZnO}$ and $\mathrm{CuO}$ nanoparticles supported mesoporous silica SBA-15", Chemical Physics Letters, v.667, pp.165-171, 2017.

[20] CHE, S., SAKAMOTO, Y., TERASAKI, O., et al., "The structure and morphology control of mesoporous silica under acidic conditions", Microporous and mesoporous materials, v.85.3, pp.207-218, 2005.

[21] TRENDAFILOVA, I., SZEGEDI, A., MIHÁLY, J., et al., "Preparation of efficient quercetin delivery system on Zn-modified mesoporous SBA-15 silica carrier", Materials Science and Engineering: C, v.73 pp.285-292, 2017.

[22] CORDEIRO, C.S., SILVA, F.R, WYPYCH, F., et al., "Catalisadores heterogêneos para a produção de monoésteres graxos (biodiesel)”, Química Nova, v. 34, n.3, pp. 477-486, 2011.

[23] RAMOS, L. P., SILVA, F. R., MANGRICH, A. S., et al., "Tecnologias de Produção de Biodiesel Ramos, LP”, Revista virtual de química, v. 3, pp. 385-405, 2011.

[24] DI SERIO, M., TESSER, R., CASALEET, L., et al., "Heterogeneous Catalysis in Biodiesel Production: The Influence of Leaching", Topics in Catalysis,, v.53, pp. 811-919, 2010.

[25] SÁDABA, I., GRANADOS, M. L., RIISAGER, A., et al., "Deactivation of solid catalysts in liquid media: The case of leaching of active sites in biomass conversion reactions", Green Chemistry, v. 17, pp.4133 $4145,2015$.

\section{ORCID}

Bruna Ezequielle Bernardes Costa

Fernando Cesário Rangel

Rosenira Serpa da Cruz https://orcid.org/ 0000-0001-9174-8820

https://orcid.org/0000-0002-1698-9664

https://orcid.org/0000-0002-2163-4737 